\title{
HIF-1a Promotes Hepatocellular Carcinoma Metastasis by Regulating Angiogenesis and Epithelial-Mesenchymal Transition
}

\section{Min Yao}

Nantong University Medical School

\section{Li Wang}

Nantong University

\section{Xiyu Chen}

Nantong University

\section{Ying Chen}

Nantong University

Jie Yang

Nantong University

\section{Defeng Wang}

Nantong University Affiliated Hospital: Affiliated Hospital of Nantong University

\section{Wei Wu}

Nantong University Affiliated Hospital: Affiliated Hospital of Nantong University dengfu yao ( $\square$ yaodf@ahnmc.com )

Medical School of Nantong University https://orcid.org/0000-0002-3448-7756

\section{Primary research}

Keywords: hepatocellular carcinoma, HIF-1a, neovascularization, dynamic model, epithelial-mesenchymal transition

Posted Date: November 17th, 2020

DOI: https://doi.org/10.21203/rs.3.rs-105342/v1

License: (c) (i) This work is licensed under a Creative Commons Attribution 4.0 International License. Read Full License 


\section{Abstract}

Background: Invasion and metastasis of hepatocellular carcinoma (HCC) still remain to be hard in medical society. However, little knowledge is known regarding the hypoxia impact in HCC with angiogenesis and epithelial-mesenchymal transition (EMT). The aims of this study were to explore the regulating roles of hypoxia-inducible factor-1a (HIF-1a) in angiogenesis and EMT of HCC.

Method: The levels of HIF-1 a, angiopoietin-2 (Ang-2), and vascular endothelial growth factor (VEGF) expression in a cohort of chronic liver diseases were detected by enzyme- linked immunosorbent assays, and their dynamic up-regulations were confirmed in model of rat hepatocyte malignant transformation. After HIF-1a gene transfected with specific miRNA, biological behaviors of HCC cells were analyzed by transwell or invasion assay; angiogenesis and EMT were analyzed at protein level by Western blot or at mRNA by quantitative real-time PCR.

Results: The levels of circulating HIF-1a, VEGF, and Ang-2 in the HCC group (145.6 $\pm 32.6 \mu \mathrm{g} / \mathrm{L}, 458.9 \pm$ $125.3 \mu \mathrm{g} / \mathrm{L}$, and $42.9 \pm 5.1 \mu \mathrm{g} / \mathrm{L})$ were significantly higher $(P<0.001)$ than those in the LC $(79.5 \pm 8.4$ $\mu \mathrm{g} / \mathrm{L}, 206.8 \pm 56.8 \mu \mathrm{g} / \mathrm{L}$, and $26.2 \pm 6.1 \mu \mathrm{g} / \mathrm{L})$ or the $\mathrm{CH}(60.1 \pm 18.8 \mu \mathrm{g} / \mathrm{L}, 178.1 \pm 85.4 \mu \mathrm{g} / \mathrm{L}$, and $21.8 \pm$ $6.9 \mu \mathrm{g} / \mathrm{L})$ group, respectively. Dynamic up-regulations of HIF-1a and angiogenic factors have been confirmed by rat model with hepatocyte malignant transformation. There were closely positive correlations $(P<0.001)$ between them of HIF-1a and VEGF or Ang-2. After HCC cells transfected with specific HIF-1a-miRNA, the levels of HIF-1a, VEGF and Ang-2 expression were significantly downregulated, with inhibiting infiltration or migration, blockading EMT with increasing E-cadherin and decreasing of snail, twist and vimentin.

Conclusions: HIF-1a over-expression could promote the metastasis or invasion of HCC via regulating neovascularization and EMT formation.

\section{Background}

Hepatocellular carcinoma (HCC) is one of the most commonly diagnosed and deadly malignant tumors worldwide [1]. HCC was still one of the most cancers in the inshore area of Yangtze River, China, due to hepatitis B virus (HBV) infection-related chronic hepatitis or liver cirrhosis [2]. There have been no more effective therapeutic options in the advanced HCC beyond sorafenib or a multi-targeted tyrosine kinase inhibitors, because hepatic hypoxia enhances cell proliferation, angiogenesis, and suppresses differentiation and apoptosis leads to resistance of transarterial chemoembolization (TACE) [3, 4]. Hypoxia-inducible factor-1 (HIF-1) is a heterodimer consisting of two subunits (a tightly regulated by changes in oxygen regimes and $\beta$ constitutively expressed), whereas HIF-1a is a master regulator of the transcriptional response of angiogenesis by multiple mechanisms such as some growth factors, tumor suppressor genes, oncogenes and formation of epithelial-mesenchymal transition (EMT), although it primarily involves protein ubiquitination $[5,6]$. Activation of HIF-1 a might regulate a repertoire of key 
angiogenic genes including VEGF [7] and Ang-2 [8]. Given HIF-1a central role in angiogenesis, some angiogenic growth factors should become prime targets for therapeutic angiogenesis of HCC $[9,10]$.

HIF-1a activation at early stages of rat hepatocyte malignant transformation has been confirmed, and HIF-1 a up-regulation might be associated with angiogenesis of HCC progression [11, 12]. Among the many regulatory factors of $\mathrm{HCC}$, the dynamic change and relevance of HIF-1a and VEGF play vital roles and have been observed after TACE of HCC patients [13]. Anti-angiogenic therapy is beneficial to HCC patients following surgical resection of tumor. However, satisfactory results have not been achieved for $\mathrm{HCC}$ because of HIF-1a over-expression might influence HCC biological behaviors and act in concert with signal pathways, and stimulate the required angiogenic growth factors endogenously [14 16]. However, HIF-1a how to mediate the molecular mechanisms of angiogenesis or EMT should be identified.

Therefore, the aims of the present study were to detect the serological levels of HIF-1a, angiogenic factors expressions in a cohort of cases with chronic liver diseases, confirm by the dynamic alterations in model of rat hepatocarcinogensis, and establish HIF-1a inactivation via specific miRNA for analyzing on effects of HCC cell proliferation, angiogenesis and EMT formation.

\section{Materials And Methods}

\section{Patients}

A cohort of 478 patients with chronic liver diseases including HCC $(n=298)$, liver cirrhosis $(L C, n=92)$, and chronic hepatitis $(\mathrm{CH}, \mathrm{n}=88)$ from the Hospital of Nantong University, China were investigated with written or verbal consent. Total 74 healthy people as normal controls (NC) were obtained from the Nantong Central Blood Bank, with negative viral markers (HBsAg, HBV-DNA, and anti-HCV antibody), normal ALT activity and liver B ultrasonic examination. Data of patients with chronic liver diseases \& normal control are shown in Table 1. Total $5 \mathrm{~mL}$ of blood from each patient or $\mathrm{d}$ healthy control were collected in the morning, and serum a-fetoprotein (AFP) and biochemistries were detected at once. Diagnostic criteria of liver cancer and hepatitis were based on the Chinese National Collaborative Cancer Research Group and Viral Hepatitis Meeting, respectively. This study was approved by the Ethics Committee of Nantong University Hospital (TDFY2013008) of China and performed in line with medical ethics of the Helsinki Declaration.

\section{Hepatocarcinogenesis model}

Rat models were approved by the guidelines of Animal Care and Use Committee of Nantong University, China. Total 48 Sprague-Dawley rats (SD) with $4 \sim 6$-wk-old that obtained from the Experimental Animal Center of Nantong University were made for hepatocarcinogenesis model in clean environment, 12-h light/dark cycle, and 55\% humidity, and schematic representation of the model and rat grouping are shown in Fig. 1. Control rats $(n=12)$ were fed normal diet, model rats $(n=36)$ was fed with containing 2acetylaminofluorene (2-AAF, $0.05 \%$, Sigma) diet, and checked every day. After rats sacrificed at different time, livers and blood were collected for analysis. Livers were used for pathology and total protein extraction. Liver pathological examination with Hematoxylin \& Eosin (H.\&E.) staining was diagnosed by 
independent pathologists, and histological grouping. Dynamic alterations of whole gene expression profiling were detected by Affymetrix GeneChip ${ }^{\circledR}$ Rat Genome 2302.0 Array (total 28,000 gene, YESLAB., Shanghai, China) and levels of VEGF, Ang-2 and HIF-1a in liver tissue supernatant and sera of rats were quantitatively detected by enzyme-linked immuno- sorbent assays.

\section{HIF-1a miRNA and constructing plasmid DNA (pDNA)}

According to HIF-1a gene (NM_001530), short hairpin-expressing pDNAs were constructed from pcDNA $^{\text {TM }}$ 6.2-GW/EmGFPmiR vector and BLOCK-iT ${ }^{T M}$ Pol II miR RNAi Expression Vector Kit (Invitrogen, USA). Targeting HIF-1a genes are as follows: 5 '-

TGCTGTAAAGCATCAGGTTCCTTCTTGTTTTGGCCACTGACTGACAAGAAG GACTGATGCTTTA-3' and 5'CCTGTAAAGCATCAGTCCTTCTTGTCAGTCAGTG GCCAAAACAAGAAGGAACCTGATGCTTTAC-3'. HIF-1a gene fragments were purified and confirmed by the MegaBACE 1000 Sequencing and Analysis System using DYEnamic ${ }^{\text {TM }}$ ET Dye Terminator Cycle Sequencing Kit (Amersham Biosciences, Little Chalfont, UK) according to manufacturer's instructions.

\section{Cell culture}

Human HCC MHCC-97H, SMMC-7721, PLC/PRF/5, Bel-7402, Hep3B, HepG2 and Bel-7404 cell lines from the Chinese Academy of Sciences (Shanghai, China) were cultured in RPMI-1640 (Gibco BRL, Gaithersburg, MD) containing 10\% FCS (Gibco BRL, USA), $2.0 \mathrm{mM} \mathrm{L-glutamine,} 100 \mathrm{U} / \mathrm{mL}$ penicillin and $100 \mu \mathrm{g} / \mathrm{mL}$ streptomycin in a constant environment $\left(37^{\circ} \mathrm{C}, 10 \% \mathrm{CO}_{2}\right.$ and $10 \%$ humidity).

In vitro transfection

Human HepG2 or Hep3B cells $\left(5 \times 10^{3}\right)$ were cultured on six-well plates for overnight incubation. They were divided into blank control (Con), negative miRNA (Neg) and miRNA (MiR) groups. The MiR or Neg group was transfected with HIF-1a miRNA or negative miRNA according to the instructions of relatedreagent kit (Roche, Germany).

\section{Cell migration or invasion assay}

Quantitative and qualitative analysis of HepG2 or Hep3B cells migration were assessed by in vitro Transwell assay with modified Boyden Chambers and Transwell-coated Matrigel membrane filter (BD Biosciences, Bedford, MA, USA). Cells $\left(5 \times 10^{3}\right)$ from Con, Neg, and MiR groups ( $n=3 /$ per group) were plated onto the upper compartment in without FBS or $10 \%$ FBS in the lower chamber as a chemoattractant. Fluorescent images of nuclear Hoechst staining $(10 \mu \mathrm{g} / \mathrm{mL})$ were captured at $24 \mathrm{~h}$ of incubation in a $5 \% \mathrm{CO}_{2}$ humidified at $37{ }^{\circ} \mathrm{C}$. Percentages of migrated cells in each group were counted from 10 random microscope fields for each sample in 3 independent experiments. For cell migration analysis, the modified Boyden Chambers without the Transwell-precoated Matrigel membrane filter in above method was performed.

\section{Quantitative real-time PCR}


Total RNA $(1 \mu \mathrm{g})$ that was extracted using Trizol reagent (MRC, Cincinnati, $\mathrm{OH})$ were reverse-transcripted into cDNA using the RevertAid ${ }^{\mathrm{TM}}$ First Strand cDNA Synthesis Kit (Fermentas, Vilnius, Lithuania). Each reaction well in a $25-\mu \mathrm{l}$ final volume contains $2 \mu \mathrm{L}$ of template DNA, $9.5 \mu \mathrm{L}$ of $\mathrm{ddH}_{2} \mathrm{O}, 12.5 \mu \mathrm{L}$ of SYBR Premix Ex Taq (TaKaRa, Japan), $0.5 \mu \mathrm{L}$ of HIF-1a forward primer: 5'-CCACTGCCACCACTGATGAA-3' (nt 2254-2273), and $0.5 \mu \mathrm{L}$ of reverse primer: 5'-TTGGTGAGGCTGTCCGACTT-3' (nt 2412-2431) to generate amplified product of $178 \mathrm{bp}$. Cycling program of qRT-PCR was 2 min at $95^{\circ} \mathrm{C}$ for hot-start enzyme activation, followed by 40 cycles of denaturation at $95^{\circ} \mathrm{C}$ for $10 \mathrm{~s}$, annealing at $60^{\circ} \mathrm{C}$ for $30 \mathrm{~s}$ and elongation at $72{ }^{\circ} \mathrm{C}$ for $45 \mathrm{~s}$ in ICycler (BIO-RAD).

\section{Western blot analysis}

Total proteins from HCC cells were lysed in RIPA buffer with protease and phosphatase inhibitors (Roche) and the concentrations were quantified with the Bicinchoninic acid Protein Assay Kit (Beyotime Institute of Biotechnology, Shanghai, China), and an equal amount of $50 \mu \mathrm{g}$ protein was separated by $10 \%$ sodium dodecyl sulfate-polyacrylamide gels electrophoresis (SDS-PAGE) and then transferred onto polyvinylidine difluoride (PVDF) membranes (Millipore, Billerica, MA, USA), blocked with $5 \%$ bovine serum albumin (BSA) in blocking buffer (Solarbio, China) for $2 \mathrm{~h}$ at room temperature, and incubated with specific primary rabbit anti-human antibodies overnight at $4{ }^{\circ} \mathrm{C}$, and $\beta$-actin (CST, USA) was used as protein loading control. The HIF-1a primary antibody was obtained from Santa Cruz (Univ-Bio., Shanghai, China) and the antibodies against Ang-2, Vimentin, E-Cadherin, Twist, and Snail were purchased from Abcam (Cambridge, MA, USA). Then the membranes were incubated with horseradish peroxidase-conjugated secondary goat anti-rabbit antibody (Abbkine, China). Detection was performed by enhanced chemiluminescence kit (Beyotime Institute of Biotech., Shanghai, China). All images were taken by the Quantity One software (Bio-Rad, Laboratories, Inc., USA).

\section{Enzyme-linked immunosorbent assay (ELISA)}

Concentrations of VEGF, Ang-2, and HIF-1 a in sera or supernatant were quantitatively detected according to the manufacturer's instructions with ELISA kits. Their levels were calculated using a standard curve generated with specific standards provided by the manufacturer with inter and intra-assay variances under $10 \%$. ELISA kits for VEGF, Ang-2 and HIF-1 a detections were purchased from the R\&D systems, Abingdon, UK; ADL Biotech Dev Co., USA; and Abcam Co., Shanghai, China, respectively.

\section{Statistical analysis}

Patients were divided into $\mathrm{HCC}, \mathrm{CH}$, and LC groups, with healthy persons as a NC group. Rat livers by pathological examination (H\&E staining) were divided into four groups of $\mathrm{rHCC}$, Pre-C, Deg, and NC. Data were expressed as mean \pm standard deviation $\left( \pm S D\right.$ ), and analyzed by SPSS19.0. Pearson $\chi^{2}$ test, ANOVA and $q$ test were performed to analyze the difference between different groups. A $P$ value $<0.05$ was considered to be statistically significant.

\section{Results}




\section{Serum VEGF, HIF-1a and Ang-2 in HCC patients}

Comparative analysis of serum HIF-1a, VEGF and Ang-2 levels in a cohort of patients with benign or malignant chronic liver diseases are shown in Table 2. The levels of VEGF, HIF-1a and Ang-2 were significantly higher $(P<0.001)$ in the HCC group than those in the NC, $\mathrm{CH}$ or $\mathrm{LC}$ group. If the cutoff values were set at over $300 \mu \mathrm{g} / \mathrm{L}$ for VEGF, $100 \mu \mathrm{g} / \mathrm{L}$ for HIF-1a or $35 \mu \mathrm{g} / \mathrm{L}$ for Ang-2, the positive rates in the HCC group $(83.6 \%, 88.9 \%$ or $88.6 \%)$ were significantly higher $(P<0.001)$ than those in the LC group $(16.3 \%$, $27.2 \%$ or $8.7 \%)$ or in the $\mathrm{CH}$ group $(13.6 \%, 2.3 \%$ or $4.6 \%)$, and none in the NC group, respectively. The circulating levels of HIF-1 a, VEGF and Ang-2 were synchronous increasing from NC, benign liver diseases to HCC progression.

\section{HIF-1a, VEGF and Ang-2 in rat hepatocarcinogenesis}

Schematic representation of rat hepatocarcinogenesis model with grouping according to the liver pathological changes (H.\&E. staining) is shown in Fig. 1. During hepato- carcinogensis, lots of relatedgenes were found with alterations (Sup-Fig. 1 and Sup-Table 1) and HIF-1a with increasing expressions in rat livers (Sup-Fig. 2) at different stages of HCC formation. The levels of HIF-1a, VEGF and Ang-2 expressions in livers (specific concentration, $\mathrm{ng} /$ per mg wet livers) or sera $(\mu \mathrm{g} / \mathrm{L})$ of rats were quantitatively investigated from normal control (NC) liver to granulose degeneration (Deg) at early stage, precancerous lesions (Pre-C) at middle stage, and rat $\mathrm{HCC}$ ( $\mathrm{rHCC}$ ) formation at later stage. The dynamic alterations of hepatic or circulating HIF-1a, VEGF and Ang-2 expressions in the different groups are shown in Table 3. No matter livers or blood, the levels of HIF-1a, VEGF and Ang-2 were dynamically upregulated during rat hepatocyte malignant transformation to accelerate the formation of new blood vessels to meet the need for oxygen, especially in the Pre-C or rHCC group.

\section{HIF-1a gene inhibited by specific miRNA}

Comparative analysis of HIF-1a expressions among different HCC cell lines by Western blotting is shown in Fig. 2. HIF-1a expressions were analyzed among the different human HCC cell lines (Fig. 2a). Both of HepG2 and Hep3B cells with the strongest expressions were compared with the average ratios from HIF$1 \mathrm{a}$ to $\beta$-actin (Fig. 2b) and were chosen for further investigation. Bath of HepG2 and Hep3B cells were successfully transfected with effective miRNA $(150 \mathrm{nmol} / \mathrm{L}$, Fig. 2c1 c3 \& d1 d3), with HIF-1a gradually decreased expressions at protein level $(P<0.001$, Fig. $2 \mathrm{c} 4 \& \mathrm{~d} 4)$, or at mRNA $\left(2^{-\triangle \triangle \mathrm{Ct}}\right)$ level at a time-dependent manner, no matter HepG2 cells $(P<0.001$, Fig. 2c5) or Hep3B cells $(P<0.001$, Fig. 2 d5).

\section{Relationship between HIF-1a and VEGF or Ang-2}

Down-regulating levels of VEGF and Ang-2 expression after intervening HIF-1amRNA transcription in HepG2 or Hep3B cells are shown in Table 4. To further estimate the effect of HIF-1a on VEGF or Ang-2, an endogenous gene product of HIF-1 a miRNA transcription activity in the culture media of HepG2 or Hep3B cells were analyzed at $24 \mathrm{~h}, 48 \mathrm{~h}$, and $72 \mathrm{~h}$ after miRNA transfection. The levels of VEGF and Ang- 2 were significantly decreased $(P<0.01)$ between the MiR group and the Con group. 


\section{inhibition of HCC biological behaviors}

The interfering HIF-1 a mRNA expression on effects of the biological behavior of HCC cells are shown in Fig. 3. After the HepG2 or Hep3B cells transfected with miRNA interfering plasmid for $48 \mathrm{~h}$ or $72 \mathrm{~h}$, the cell proliferation rates were significantly lower than that of the negative or control group (Fig. 3a), with the longer time and the higher inhibition rate (Fig. 3b), with lowest the clone formation number of HCC cells (Fig. 3c1). After the HCC cells transfected with miRNA interfering plasmid for $72 \mathrm{~h}$, the migration capacity of HCC cells in the miRNA group were significantly lower $(P<0.001$, Fig. 3d) than those of the control or negative group. Also, the ability of HCC cells invasiveness in the miRNA group were significantly lower $(P$ $<0.001$, Fig. 3e) than those of the control or negative group, indicated that interfering with HIF-1 gene transcription could significantly affect the biological behaviors of HCC cells, especially in migration and invasiveness.

\section{HIF-1a activation promotes EMT formation}

Interfering HIF-1a gene transcription of HCC cells on effects of EMT-related protein expressions are shown in Fig. 4. Alterations of EMT-related epithelial marker (E-cadherin), mesenchymal indicator (Vimentin), transcriptional factor (Snail \& Twist) levels in the culture medium of HCC cells were analyzed by Western blot analysis. There were no significant differences of those proteins between the Neg group and the Con group. when HepG2 cells $(a \sim d)$ or Hep3B cells $(e \sim f)$ with stable silencing HIF-1a transcription in the MiR group, the levels of the E-cadherin were significantly $(P<0.001)$ increasing than that in the NC group (Fig. 4a, a1\& Fig. 4e, e1); otherwise the levels of Vimentin ( $P<0.001$, Fig. 4b, b1\& Fig. 4f, f1), and Snail ( $P$ $<0.001$, Fig. 4c, c1 \& Fig. 4g, g1) and Twist ( $P<0.001$, Fig. 4d, d1 \& Fig. 4h, h1) were significantly lower less than those in the NC group.

The expressions of EMT-related proteins in HCC cells were significantly inhibited by interfering with HIF1a mRNA except of E-cadherin, and it should be a novel regulating mechanism (Fig. 5) insight into the invasion and metastasis of HCC is by decreasing E-cadherin or increasing Vimentin, Twist and Snail signaling for molecular-targeted therapy. HIF-1a over-expression could promote the proliferation of cancer cells, EMT, and angiogenic factor expressions for HCC metastasis.

\section{Discussion}

$\mathrm{HCC}$ is one of the most common malignant cancers worldwide and a high malignancy solid tumor with hypoxia microenvironment, high-vascular, and traditional-therapy resistance [16]. HIF-1a is a key transcription regulator for many genes and promotes tumorigenicity by up-regulating its target genes, which were involved in apoptosis, proliferation, angiogenesis, invasion and metastasis of HCC such as VEGF, Ang-2, Wnt3a, IGF-II, and so on[17]. This study has investigated the relationship between HIF-1a and HCC-related angiogenesis or EMT, and demonstrated that abnormalities of HIF-1a, Ang-2 and VEGF expressions in $\mathrm{HCC}$, the up-regulating expression in rat hepatocarcinogenesis, and the acting mechanism 
of HIF-1 a were confirmed on effects of the proliferation, angiogenesis and EMT of HCC cells with specific miRNA transfection.

Maintenance appropriate oxygen and angiogenic factors are essential for HCC growth. HIF-1a has been implicated in regulating hepatic inflammatory progression, and associated with multiple inflammationrelated cancers or signaling pathway activation of HBV-related HCC [18]. The presence of hypoxia in tissues restricts HCC overgrowth because of HIF-1 a regulating cellular metabolism, immune escape, angiogenesis, metastasis, extracellular matrix remodeling, cancer stem cells (CSC) [19] or MDR via regulating PI3K/AKT/HIF-1a/MDR-1 pathway [20]. HIF-1a-deficient HCC cells displayed significantly reduced anchorage-independent growth and enhanced sensitivity toward chemotherapy [4]. Angiogenesis is thought to depend on a perfectly coordinated balance between endogenous-positive and negative regulatory factors. The investigation of HIF-1a, VEGF and Ang-2 levels in sera of patients with benign or malignant chronic liver diseases suggested that their synchronous increasing expression from normal control, chronic hepatitis, liver cirrhosis to HCC [21].

Abnormalities of HIF-1 $a$ and angiogenic factors were associated with rat hepatocarcinogenesis [22]. HIF$1 \mathrm{a}$ binds to an evolutionary conserved HRE located in the first introns of Ang-2. All of the HIF-1a, VEGF and Ang-2 expressions demonstrated a tendency to increase with the histopathological changes: HCC more than precancerosis more than degeneration more than controls. Their levels were markedly higher in the rHCC and precancerous groups than those in the degeneration or control group. Blood levels of HIF$1 a$, VEGF and Ang-2 expressions in the HCC or precancerous group were higher than in the degeneration or control group. There was an apparent positive correlation between in blood and livers. HIF-1a overexpression was confirmed during hepatocytes malignant transformation with HCC progress, because it regulates the transcription of downstream numerous genes including angiogenesis or proliferation to set basis for HCC growth and metastasis [23]. However, the specific miRNA decreased HIF-1a expression and suppressed angiogenesis in the HCC cell lines by down- regulating VEGF and Ang-2, indicated that HIF-1a regulate the angiogenesis of $\mathrm{HCC}$.

EMT is a cellular programmed that is known to be crucial for malignant progression that increases the HCC invasive and metastasis potential with increasing expression of mesenchymal indicators or transcription factors and down-regulating epithelial marker levels [24]. In the context of liver neoplasias, EMT confers on cancer cells promoting tumor-initiating and easily metastatic potential, and more resistance to elimination by therapeutic regimens for HCC. Vimentin might increase the migration and invasiveness of $\mathrm{HCC}$ and relate to reduce E-cadherin and up-regulate $\mathrm{N}$-cadherin, while increased vimentin was associated with poor prognosis of HCC. In this study, the relationship between the EMT-related biomarkers (E-cadherin, Vimentin, Snail, and Twist) and HIF-1 a were investigated at cell level. After the HepG2 or Hep3B cells with stable silencing HIF-1a in the MiR group compared with the CN or Neg group, the E-cadherin at protein level was significantly un-regulation, but the levels of Vimentin, Snail, and Twist were markedly down-regulating expression, suggesting that HIF-1 a promote HCC metastasis by increasing EMT ability and as a potential molecular target for HCC therapy. 


\section{Conclusions}

Our data provide novel evidence that HIF-1a activation could promote metastasis and invasion of HCC via regulating angiogenesis and EMT-related proteins. It should provide a regulating mechanism insight into HCC metastasis. From silencing HIF-1 a gene transcription could inhibit the metastasis with angiogenesis or EMT formation of HCC, and had got some associations between HIF-1a and metastasis. However, the exact mechanisms were needed to be confirmed with more studies in vitro and in vivo. Further work should be done how to application of HIF-1 a miRNA plus multi-targeting strategies for HCC effective therapy.

\section{Abbreviations}

AFP: Alpha-fetoprotein; Ang-2: Angiopoietin-2; CH: Chronic hepatitis; ELISA: Enzyme -linked immunosorbent assay; EMT: Epithelial-mesenchymal transition; HBV: Hepatitis B virus; HCC: Hepatocellular carcinoma; HCV: Hepatitis C virus; H.\&E: Hematoxylin \& Eosin staining; HIF-1a: Hypoxia inducible factor-1a; IHC: Immunohistochemistry; LC: Liver cirrhosis: RT-Q-PCR: Real-time quantitativepolymerase chain reaction; SDS-PAGE: Sodium dodecyl sulfate-polyacrylamide gels electrophoresis; TACE: Trans- arterial chemoembolization; VEGF: Vascular endothelial growth factor.

\section{Declarations}

Authors' contributions: MY, LW and XYC conceived the study, analyzed and interpreted the data. MY, LW, $X Y C, Y C$ and JY performed the experiments. LW and DFW performed statistical analysis and bioinformatics. MY and WW acquired material and data. MY, LW and DFY acquired the funding. MY, LW and DFY wrote the manuscript. All authors have read and approved the final manuscript.

Author details: ${ }^{1}$ Department of Immunology, Medical School of Nantong University; ${ }^{2}$ Research Center of Clinical Medicine, Affiliated Hospital of Nantong University; ${ }^{3}$ Department of Medical Informatics, Medical School of Nantong University; ${ }^{4}$ Research Center for Intelligence Information Technology of Nantong University; ${ }^{5}$ Department of Molecular Biology, Life Science School of Nantong University, Jiangsu Province 226019, China.

Acknowledgements: No applicable.

Competing interests: The authors declare that they have no competing interests.

Availability of data and materials: The datasets used and /or analyzed during the current study are available from the corresponding author on reasonable request.

Consent for publication: Not applicable. 
Ethics approval and consent to participate: All procedures conformed to the Helsinki Declaration for the research on humans. Signed informed consent was obtained from all patients and the experimental research has been performed with the ethical approval provided (TDFY2013008) by the Affiliated Hospital of Nantong University, China and the study of rat models were approved by the guidelines of Animal Care and Use Committee of Nantong University, China.

Funding: This study was supported by the Projects of National Natural Science Foundation (81673241, 31872738 , and 81873915) of China, and the Ministry of Science \& Technology National Key Research \& Development Program (2018YFC0116902) of China

\section{References}

1. Jindal A, Thadi A, Shailubhai K. Hepatocellular carcinoma: etiology and current and future drugs. J Clin Exp Hepatol. 2019;9(2):221-32.

2. Chen JG, Zhu J, Zhang YH, Zhang YX, Yao DF, Chen YS, et al. Cancer survival in Qidong between 1972 and 2011: A population-based analysis. Mol Clin Oncol. 2017;6(6):944-54.

3. Lin D, Wu J. Hypoxia inducible factor in hepatocellular carcinoma: A therapeutic target. World J Gastroenterol. 2015;21(42):12171-8.

4. Méndez-Blanco C, Fondevila F, García-Palomo A, González-Gallego J, Mauriz JL. Sorafenib resistance in hepatocarcinoma: role of hypoxia-inducible factors. Exp Mol Med. 2018;50(10):1-9.

5. Keith B, Johnson RS, Simon MC. HIF1a and HIF2a: sibling rivalry in hypoxic tumour growth and progression. Nat Rev Cancer. 2011;12(1):9-22.

6. Jing L, Ruan Z, Sun H, Li Q, Han L, Huang LX, et al. Epithelial-mesenchymal transition induced cancer- stem-cell-like characteristics in hepatocellular carcinoma. J Cell Physiol. 2019;234(10):18448-58.

7. Li S, Yao D, Wang L, Wu W, Qiu L, Yao M, et al. Expression characteristics of hypoxia-inducible factor$1 \mathrm{a}$ and its clinical values in diagnosis and prognosis of hepatocellular carcinoma. Hepat Mon. 2011;11(10):821-8.

8. Morse MA, Sun W, Kim R, He AR, Abada PB, Mynderse M, et al. The role of angiogenesis in hepatocellular carcinoma. Clin Cancer Res. 2019;25(3):912-20.

9. Petrillo M, Patella F, Pesapane F, Suter MB, lerardi AM, Angileri SA, et al. Hypoxia and tumor angiogenesis in the era of hepatocellular carcinoma transarterial loco-regional treatments. Future Oncol. 2018;14(28):2957-67.

10. Vogel A, Saborowski A. Current strategies for the treatment of intermediate and advanced hepatocellular carcinoma. Cancer Treat Rev. 2019;82:101946.

11. Yao DF, Jiang H, Yao M, Li YM, Gu WJ, Shen YC, et al. Quantitative analysis of hepatic hypoxiainducible factor-1 alpha and its abnormal gene expression during the formation of hepato- cellular carcinoma. Hepatobiliary Pancreat Dis Int. 2009;8(4):407-13. 
12. Tirpe AA, Gulei D, Ciortea SM, Crivii C, Berindan-Neagoe I. Hypoxia: overview on mypoxia-mediated mechanisms with a focus on the role of HIF genes. Int J Mol Sci. 2019;20(24):6140.

13. Liu K, Min XL, Peng J, Yang K, Yang L, Zhang XM. The changes of HIF-1a and VEGF expression after TACE in patients with hepatocellular carcinoma. J Clin Med Res. 2016;8(4):297-302.

14. Mortezaee K. Hypoxia induces core-to-edge transition of progressive tumoral cells: A critical review on differential yet corroborative roles for HIF-1a and HIF-2a. Life Sci. 2019;242:117145.

15. Chen Y, Huang F, Deng L, Yuan XW, Tao Q, Wang TL, et al. HIF-1-miR-219- SMC4 regulatory pathway promoting proliferation and migration of HCC under hypoxic condition. Biomed Res Int. 2019; 2019:8983704.

16. Yao D, Jiang D, Huang Z, Lu J, Tao Q, Yu Z, et al. Abnormal expression of hepatoma specific gammaglutamyl transferase and alteration of gamma-glutamyl transferase gene methylation status in patients with hepatocellular carcinoma. Cancer. 2000;88(4):761-9.

17. Tai BJ, Yao M, Zheng WJ, Shen YC, Wang L, Sun JY, et al. Alteration of oncogenic IGF-II gene methylation status associates with hepatocyte malignant transformation. Hepatobiliary Pancreat Dis Int. 2019;18(2):158-63.

18. Yang SL, Ren QG, Zhang T, Pan XL, Wen L, Hu JL, et al. Hepatitis B virus X protein and hypoxiainducible factor-1 a stimulate Notch gene expression in liver cancer cells. Oncol Rep. 2017;37(1):348-56.

19. Cui CP, Wong CC, Kai AK, Ho DWH, Lau EYT, Tsui YM, et al. SENP1 promotes hypoxia-induced cancer stemness by HIF-1a deSUMOylation and SENP1/HIF-1a positive feedback loop. Gut. 2017;66(12):2149-59.

20. Xie Y, Zhong DW. AEG-1 is associated with hypoxia-induced hepatocellular carcinoma chemoresistance via regulating PI3K/AKT/HIF-1alpha/MDR-1 pathway. EXCLI J. 2016;15:745-57.

21. Wang D, Zhang X, Lu Y, Wang X, Zhu L. Hypoxia inducible factor 1a in hepatocellular carcinoma with cirrhosis: Association with prognosis. Pathol Res Pract. 2018;214(12):1987-92.

22. Zhao H, Yao P, Fu N, Chen L. deSUMOylation signaling: a novel mechanism of liver CSC properties and hepatocarcinogenesis in hypoxia. Acta Biochim Biophys Sin. 2017;49(12):1135-7.

23. Matsuura Y, Wada H, Eguchi H, Gotoh K, Kobayashi S, Kinoshita M, et al. Exosomal miR-155 derived from hepatocellular carcinoma cells under hypoxia promotes angiogenesis in endothelial cells. Dig Dis Sci. 2019;64(3):792-802.

24. Dongre A, Weinberg RA. New insights into the mechanisms of epithelial-mesen- chymal transition and implications for cancer. Nat Rev Mol Cell Biol. 2019;20(1):69-84.

\section{Tables}

Due to technical limitations, table 1 to 4 is only available as a download in the Supplemental Files section. 


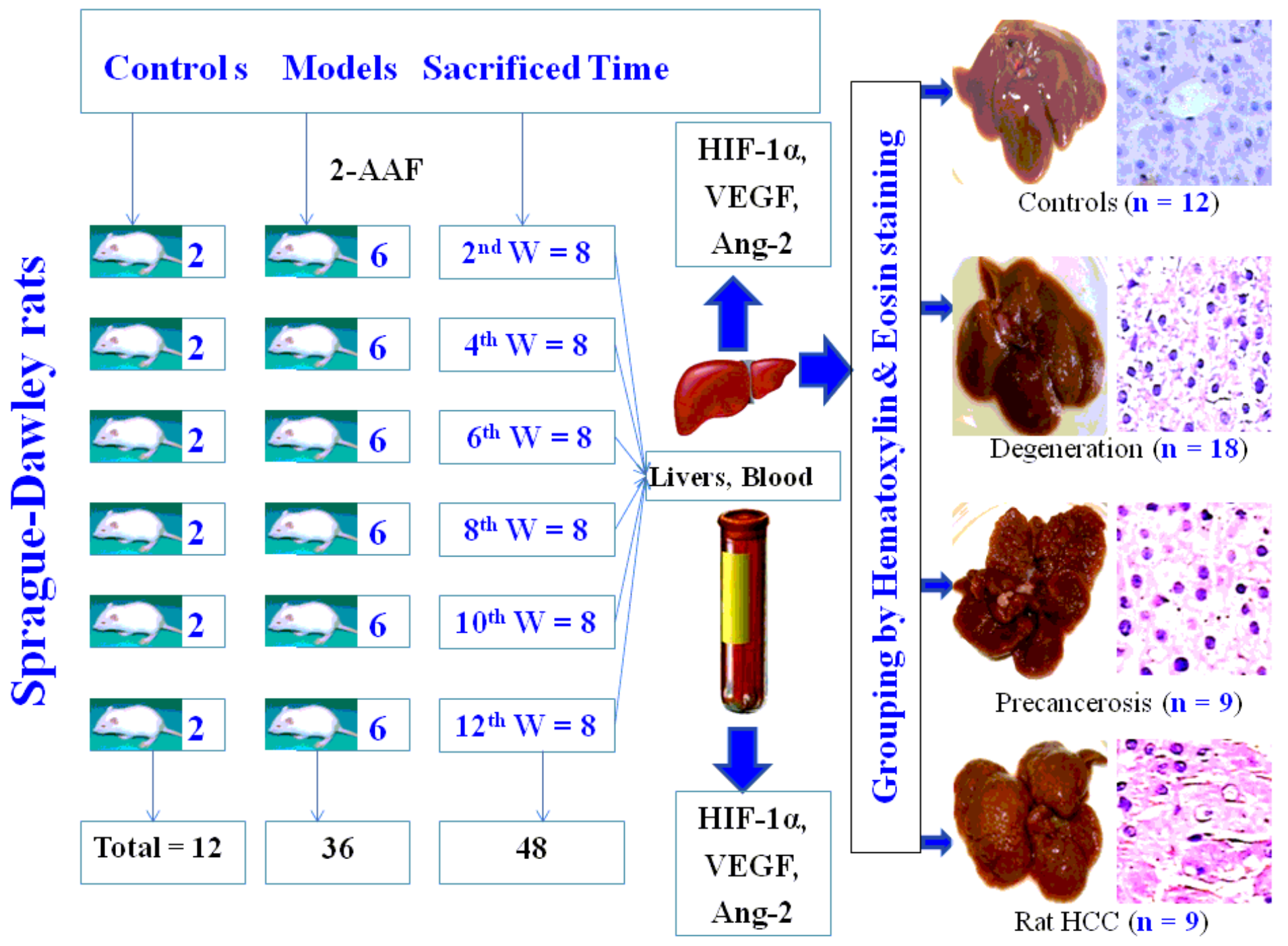

Figure 1

Schematic representation of rat hepatocarcinogenesis and grouping Dynamic models of Sprague-Dawley (SD) rats were made with 2-acetylaminofluorene (2-AAF, Sigma, USA) inducing. All rats according to the morphological changes of liver sections were examined by the Hematoxylin \& Eosin staining $(\times 200)$, and divided into four groups: hepatocyte degeneration $(n=18)$, precancerosis $(n=9)$, rat $\operatorname{HCC}(n=9)$, and control $(n=12)$. Ang-2, angiopoietin-2; HIF-1a, hypoxia-inducible factor- $1 a$; and VEGF, vascular endothelial growth factor 


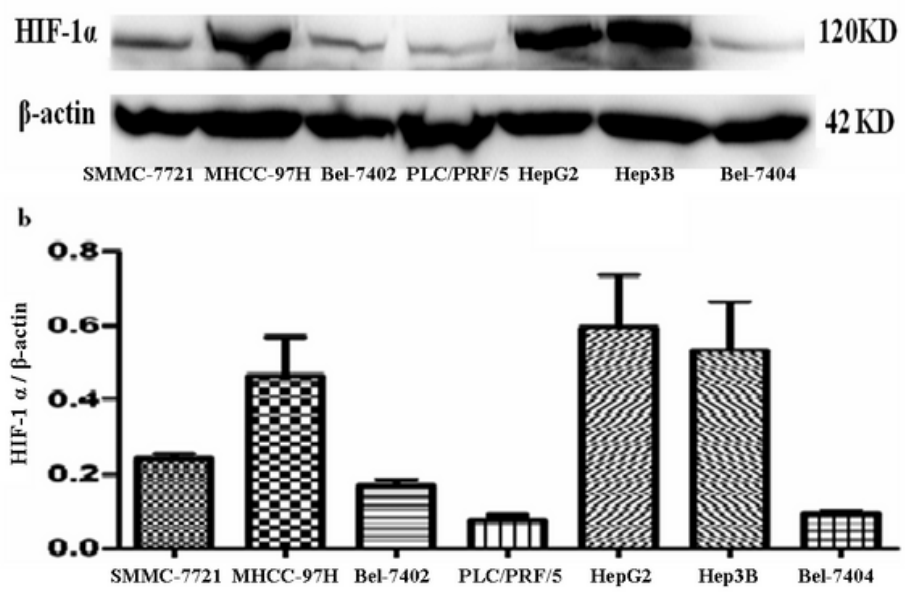

c HepG2 cells
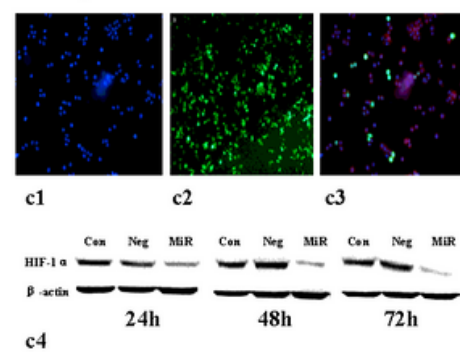

c4
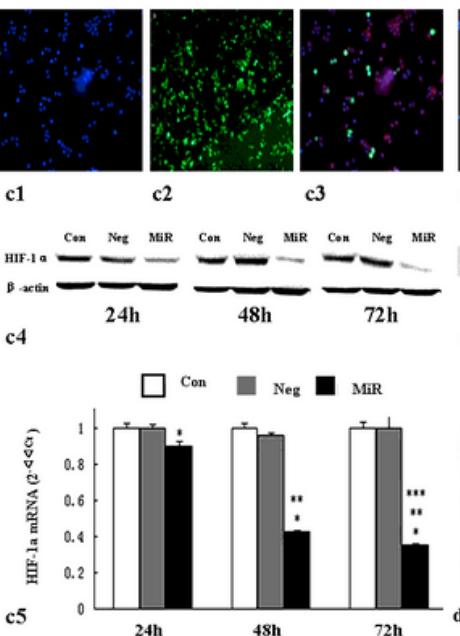

d Hep3B cells

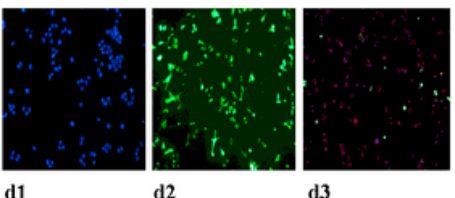

d3
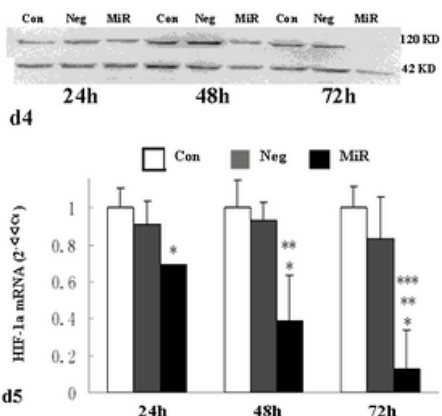

\section{Figure 2}

HIF-1a in HCC cells and inhibition by specific miRNA HCC cell lines: a, HIF-1a in SMMC-7721, MHCC-97H, Bel-7402, PLC/PRF/5, HepG2, Hep3B, and Bel-7404 cells; $b$, the average ratios ( \pm SD, $n=5$ ) of HIF-1a to $\beta$ actin from left to right corresponding to above HCC cells analyzed by the QuantityOne Program (Bio-Rad, USA); c, HepG2 \& d, Hep3B cells (magnification $\times 40$ ); c1 \& d1, the micrograph (no fluorescence) in the Con group at $24 \mathrm{~h} ; \mathrm{c} 2 \&$ d2, the fluorescence micrograph at $24 \mathrm{~h}$, or c3 \& d3, at $72 \mathrm{~h}$; c4 \& d4, the downregulating of HIF-1a (up, $120 \mathrm{kD}$ ) at protein level in the miR group depended on time confirmed by Western blotting, with $\beta$-actin (down, 42 kD) as control; c5 \& d5, the Quantitative analysis of HIF-1a mRNA after miRNA (150 mol/L) transfection, and HIF-1a gene copies were down-regulated at a time- dependent manner by real-time quantitative PCR. Con, the Con group; Neg, the Neg group; MiR, the mMiR group. ${ }^{*} \mathrm{P}<$ 0.05 , compared with the Con group; ${ }^{*} \mathrm{P}<0.001$ or $* \star \star \mathrm{P}<0.001$, compared with the miR group at $24 \mathrm{~h}$ or $48 \mathrm{~h}$. 
a

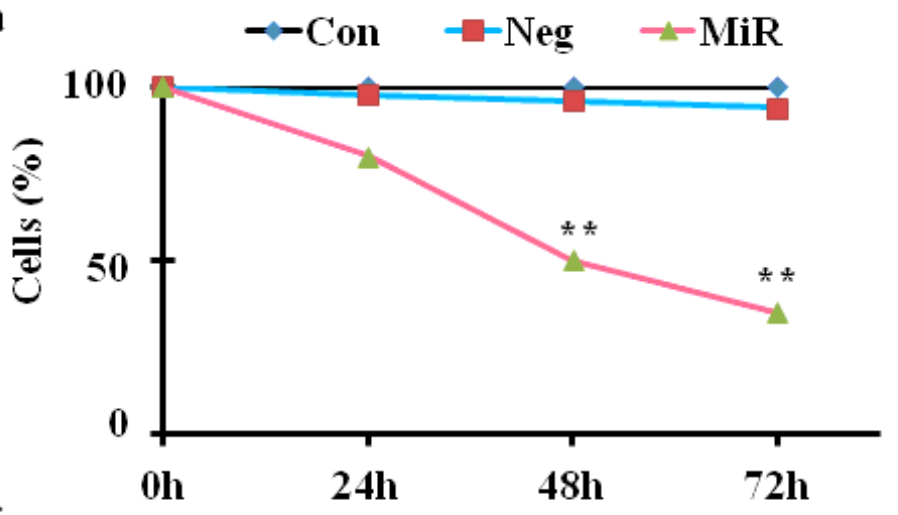

c
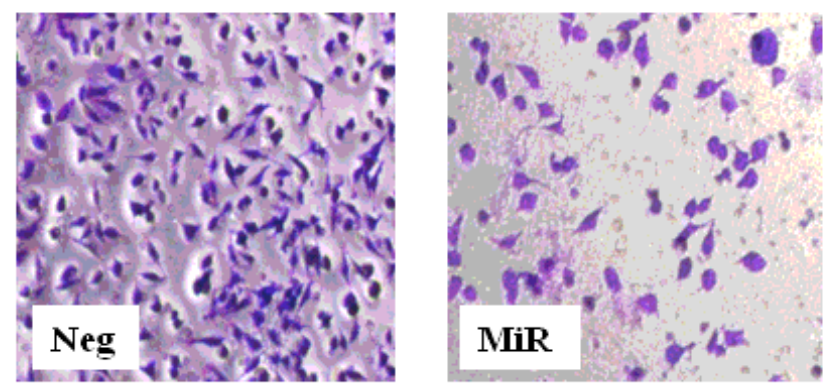

e

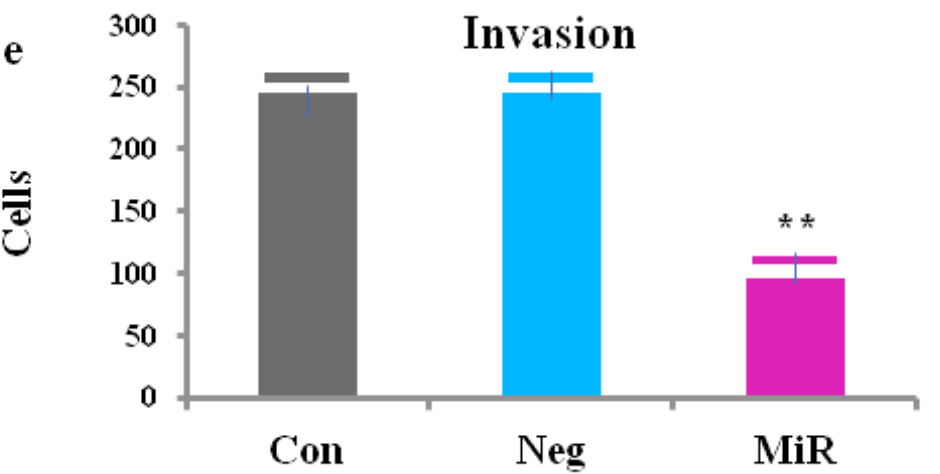

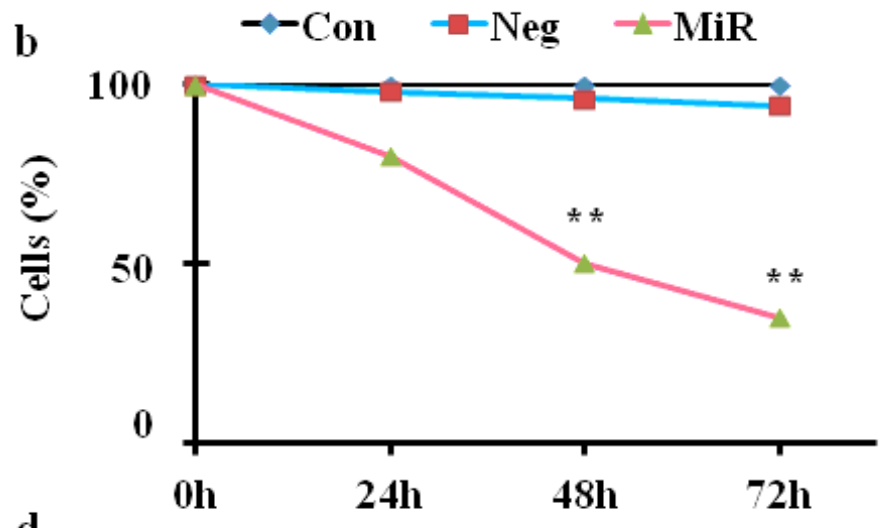

d
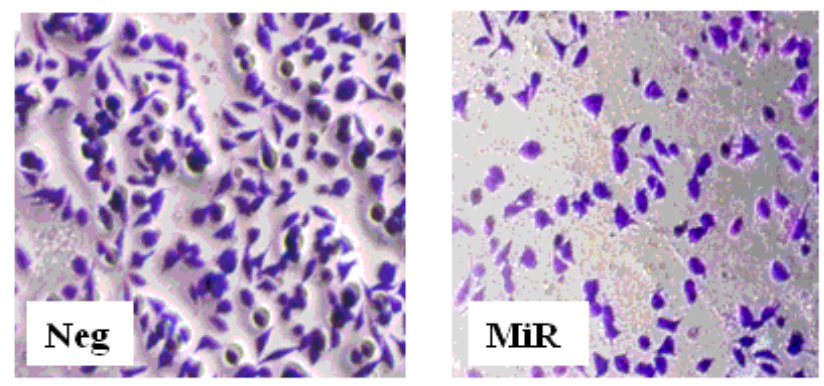

Metastasis

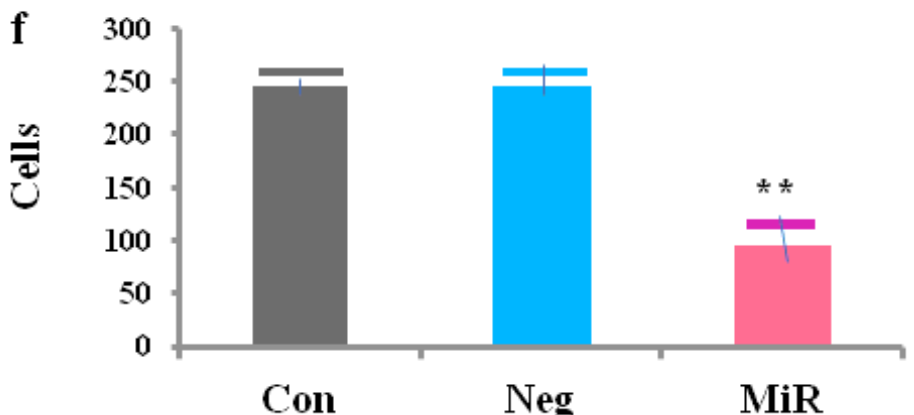

Figure 3

HIF-1a inhibiting with cell biological behaviors Interfering HIF-1a mRNA affected significantly biological behaviors of HCC cells. a, the proliferation inhibiting curve of HepG2 cells; $b$, the proliferation inhibiting curve of Hep3B cells; c, the crystal violet staining of HepG2 cells (magnification $\times 200$ ) in the miR or Neg group; $d$, the crystal violet staining of Hep3B cells (magnification $\times 200$ ) in the miR group and the Neg group; e, the number of HepG2 cell migration; $f$, the number of Hep3B cell invasion. ${ }^{*} \mathrm{P}<0.05$ or ${ }^{*} \mathrm{P}<$ 0.001 , compared with the Con group. 

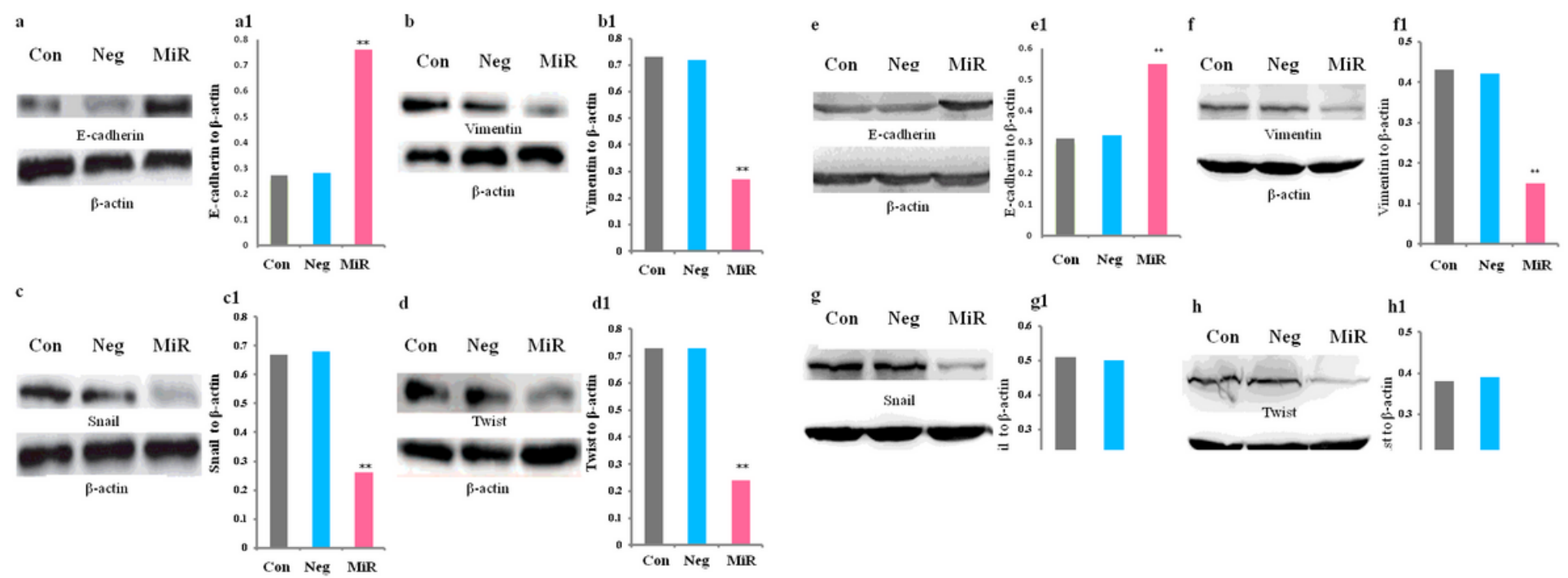

Figure 4

Silencing HIF-1a with EMT of HCC cells Epithelial or mesenchymal biomarker and transcriptional factors were involved in HCC metastasis with EMT. a d, HepG2 cells; e f, Hep3B cells; After silencing HIF-1a gene, the related-proteins were analyzed by the Western blotting with $\beta$-actin as loading control. a \& e, Ecadherin, and a $1 \&$ e1, the relative ratio $(n=6) ; b \& f$, vimentin, and $b 1 \& f 1$, the relative ratio $(n=6) ; c \& g$, snail, and $\mathrm{c} 1 \& \mathrm{~g} 1$, the relative ratio $(n=6) ; \mathrm{d} \& \mathrm{~h}$, twist, and $\mathrm{d} 1 \& \mathrm{~h} 1$, the relative ratio $(n=6)$. Con, the Con group; Neg, the Neg group; MiR, the MiR group. ${ }^{*} \mathrm{P}<0.05$ or ${ }^{\star} \mathrm{P}<0.001$, compared with the Con group. 


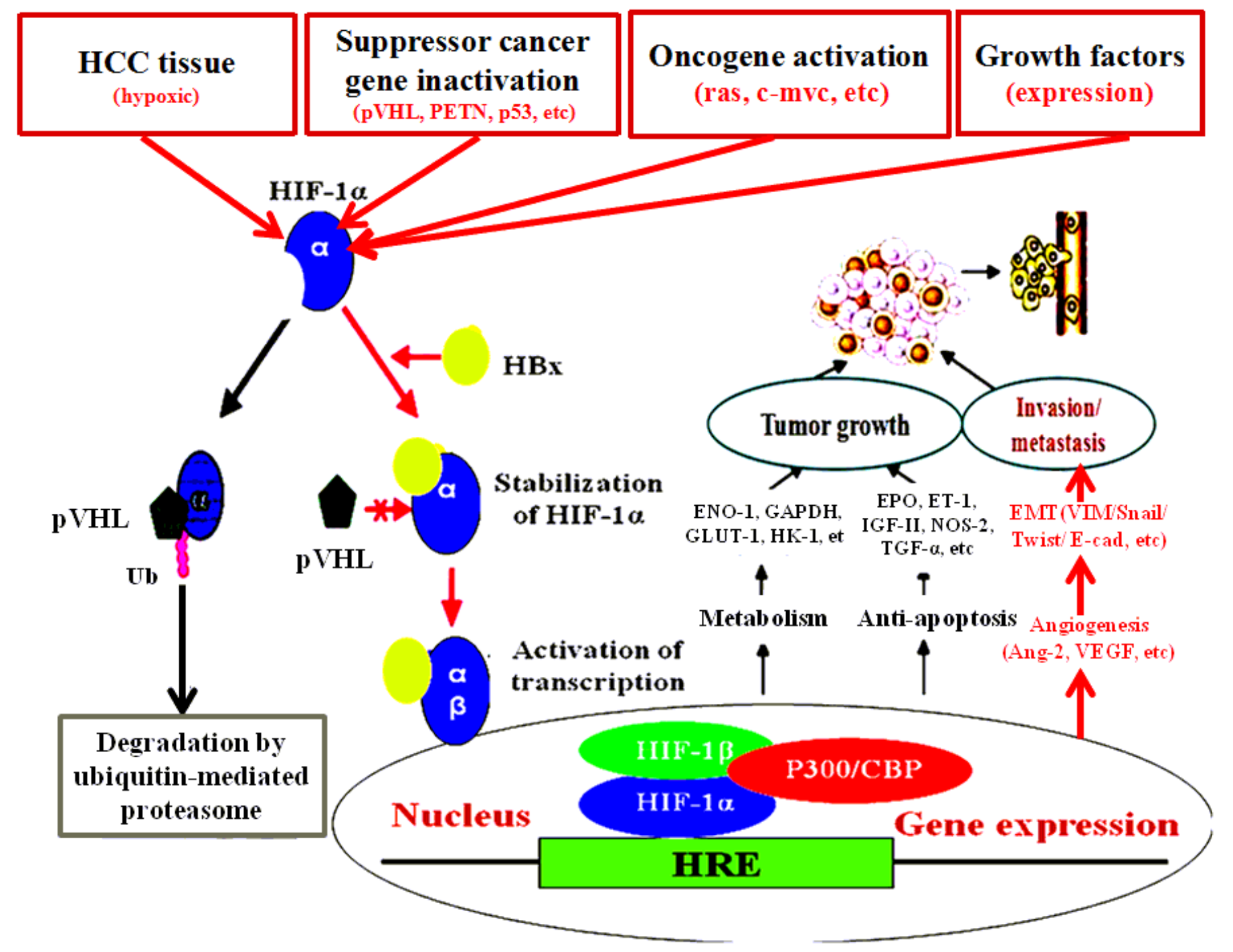

Figure 5

Possible mechanism of HIF-1a promoting HCC metastasis HIF-1a facilitating proliferation, angiogenic factors and EMT of HCC cells could be a new mechanism insight into metastasis that could be inhibited by down-regulating Ang-2, VEGF and E-cadherin or up-regulating vimentin, twist, and snail. enolase 1 (ENO-1); erythro- poietin (EPO); endothelin-1 (ET-1); glyceraldehydes-3-phosphate dehydrogenase (GAPDH); glucose transporter 1 (GLUT-1); hexokinase-1 (HK-1); hypoxia response element (HRE); insulinlike growth factor-II (IGF-II); mechanistic target of rapamycin kinase (mTOR); nitric oxide synthase-2 (NOS2); transforming growth factor-a (TGF-a); and von Hippel-Lidau (VHL).

\section{Supplementary Files}

This is a list of supplementary files associated with this preprint. Click to download.

- YMTabs.doc

- YMsuppfigs.docx 